TRABAJOS ORIGINALES

\title{
Begonia ludwigii y B. parcifolia (Begoniaceae) dos registros nuevos para la flora peruana
}

\section{Begonia ludwigii and B. parcifolia (Begoniaceae) two new records to the Peruvian flora}

\section{Boris Esquerre-Ibañez 1* y Mark C. Tebbitt 2}

1 Universidad Nacional Pedro Ruiz Gallo, Ciudad Universitaria, Juan XXIII 391, Lambayeque, Perú.

2 Department of Biological and Environmental Sciences, California University of Pennsylvania, California, PA 15419-1394, U.S.A.

${ }^{*}$ Autor para correspondencia

Email Boris Esquerre-Ibañez: kamijo002@gmail.com

Email Mark Tebbitt: tebbitt@calu.edu

\section{Resumen}

Begonia ludwigii Irmscher y B. parcifolia C. DC. se registran para la flora peruana, donde ocurren dentro de la zona biodiversa Amotape-Huancabamba. Ambas especies fueron originalmente consideradas como endémicas de Ecuador. Dentro de Perú, B. Iudwigii es reportada de cuatro poblaciones en los Departamentos de Piura, Lambayeque y Cajamarca, mientras que B. parcifolia es reportada de tres poblaciones en los Departamentos de Tumbes, Piura y Cajamarca. Se proporcionan descripciones de B. ludwigii y B. parcifolia y se mapean y discuten sus distribuciones. También se presenta información sobre su taxonomía y estado de conservación.

Palabras clave: Begonia; Begoniaceae; Begonia Sección Knesebeckia; Ecuador; Perú

\section{Abstract}

Begonia Iudwigii Irmscher and B. parcifolia C. DC. are newly recorded for the Peruvian flora, where they occur within the biodiverse Amotape-Huancabamba zone. Both species were originally considered to be endemic to Ecuador. Within Peru, B. Iudwigii is reported from four populations in the Departments of Piura, Lambayeque and Cajamarca, while $B$. parcifolia is reported from three populations in the Departments of Tumbes, Piura and Cajamarca. Descriptions of $B$. Iudwigii and $B$. parcifolia are provided and their distributions mapped and discussed. Information about their taxonomy and conservation status are also presented.

Keywords: Begonia; Begoniaceae; Begonia Section Knesebeckia; Ecuador; Peru

Citación:

Esquerre-Ibañez B. \& M.C. Tebbitt. 2018. Begonia ludwigii Irmscher y B. parcifolia C. DC. (Begoniaceae) dos registros nuevos para la flora peruana. Revista peruana de biología 25(4): 437 - 444 (Noviembre 2018) doi: http://dx.doi.org/10.15381/rpb.v25i4.15535

$\begin{array}{ll}\text { Presentado: } & 04 / 05 / 2018 \\ \text { Aceptado: } & 14 / 11 / 2018 \\ \text { Publicado online: } & 07 / 12 / 2018\end{array}$

Información sobre los autores:

$\mathrm{BE}-\mathrm{I}$ y $\mathrm{MCT}$, realizaron las colectas, analizaron los datos, redactaron revisaron y aprobaron el manuscrito.

Los autores no incurren en conflictos de intereses.

Permisos de colecta:

Las colectas de Begonia ludwigii están autorizadas por SERFOR mediante Resolución N²47-2016-SERFOR/DGGSPFFS y B. parcifolia mediante Resolución Nº 077-2014 SERFOR/DGGSPFFS. 


\section{Introducción}

El género Begonia comprende especies tropicales terrestres, las cuales pueden ser acaules, con tallo tipo cańa, tallo suculento, herbáceo, arbustivo o trepador, raramente epífitas, con tubérculos generalmente cuando son especies dormantes (Doorenbos et al. 1998, Tebbitt 2005). La importancia comercial de este género, por un lado se debe a que es reconocido a nivel mundial por su potencial como planta ornamental, por la belleza de sus hojas y flores, por otro lado también es usada tradicionalmente como alimento y medicina (Rajbhandary 2013).

En el Perú se han reportado 76 especies y 38 endemismos (Brako \& Zarucchi 1993, León et al. 2006) de este género, cuya distribución es abundante en la zona boscosa andina y amazónica. En recientes años, se han ido descubriendo nuevos taxones (Tebbitt 2011, Tebbitt 2015, Tebbitt 2016, Moonlight \& Tebbitt 2016, Moonlight et al. 2017) colocando a Perú como el tercer país con mayor diversidad de Begonia en las Américas después de Brasil y Colombia (Moonlight et al. 2017).

Las recientes colecciones de material botánico en el norte de Perú y revisiones de material de herbario están permitiendo descubrir nuevos taxones, así como ańadir nuevos registros a la flora ya conocida en Begonia, ampliando la distribución de algunas especies que antes se consideraban endémicas y muy aisladas.

Las dos especies examinadas aquí, $B$. ludwigii y $B$. parcifolia, fueron consideradas estrictamente endémicas restringidas al sur de Ecuador, pero nuestra investigación de campo ha demostrado que están más extendidas que lo reportado anteriormente y que ocurren en el norte de Perú, además de Ecuador.

\section{Material y métodos}

El material comprende las colecciones llevadas a cabo por los autores en los departamentos de Lambayeque: distritos Salas e Inkawasi, Piura: distritos Ayabaca y Canchaque, y Cajamarca: distrito La Florida, en el Norte del Perú, y las colecciones de herbario consultadas en: AAU, E, HUT, MO, MOL, QCA, QCNE, S, SEL, US y USM.

La metodología consistió en la búsqueda del material en campo, donde se tomaron los datos de georreferencia, hábitat, detalles de la planta, fotografías y posteriormente la colecta de material para ser prensado siguiendo el procedimiento clásico (Rodríguez \& Rojas 2016) y parte del material se conservó fresco y turgente en bolsas "ziploc", papel húmedo y cajas "teknopor", para su evaluación descriptiva bajo estereomicroscopio en el Laboratorio de Cultivo de Tejidos Vegetales de la Universidad Nacional Pedro Ruiz Gallo, Lambayeque, Perú.

Se contrastó con literatura (Brako \& Zarucchi 1993, Ulloa et al. 2004) y bases de datos (TROPICOS 2018) que B. ludwigii y $B$. parcifolia no han sido reportadas para el territorio peruano, a excepción de una breve mención de una población peruana de $B$. ludwigii en una publicación reciente (Tebbitt 2017) por lo que esta investigación pretende formalizar y también divulgar más ampliamente la distribución de estas dos especies, previamente solo reportadas para Ecuador, ahora como nuevos miembros integrantes de la flora peruana.

\section{Resultados y discusión}

\section{Begonia ludwigii Irmscher}

(Figs. 1-3)

Bibliotheca Botanica 116: 113. 1937

Tipo: ECUADOR, provincia de Chimborazo, valle del río Chanchan, Naranjapata, 530 m, 25 setiembre 1933. Diels 1204 (holotipo B!; fototipo US!).

Begonia compacticaulis Irmsch., Bot. Jahrb. Syst. 74: 612. 1949. - Tipo: Ecuador, Prov. Chimborazo, Huigra, julio 1923, A.S. Hitchcock 20315 (holotipo US!; isotipo NY!).

Begonia ecuadoriensis auct. non Buxton: hort. ex Everett, J. New York Bot. Gard. 51: 4. 1940.

Begonia griseocaulis auct. non Irmsch.: L.B.Sm. \&

B.G.Schub., Mem. New York Bot. Gard. 8: 38. 1952.

Begonia rigida auct. non Linden ex Regel: A.Clarke, The Begonian 14: 150.1947 .

Hierba caulescente, de tallo suculento, grueso, con cicatrices peciolares donde también asoman las yemas foliares, glabro. Estípulas caducas, ovado-oblongas, $10-12 \times 4,5 \mathrm{~mm}$, con el ápice suavemente agudo, glabras. Peciolo de hasta $16 \mathrm{~cm}$ de largo, con tricomas esparcidos, a veces con la base rojiza, y una hilera de tricomas prominentes de 5-8 mm de largo en la unión con la lámina, blanquecinos, esta zona de la unión con la lámina a veces matizada de rojo, también el ápice de la hilera de tricomas puede ser rojizo. Lámina membranácea, con tricomas, partida en 5-7 lóbulos asimétricos, de borde inciso, generalmente verdosa, matizada de rojo vinoso a marrón mayormente cuando

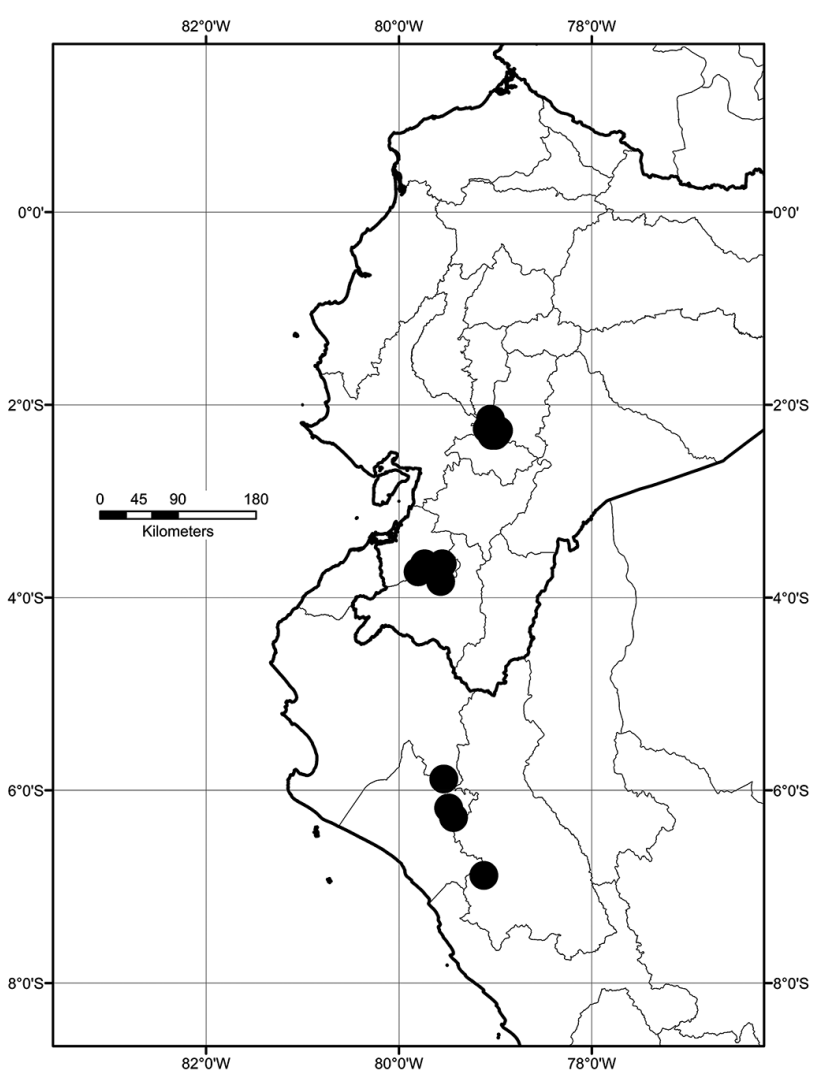

Figura 1. Distribución de Begonia ludwigii en Ecuador y Perú. 
son juveniles en la cara abaxial y adaxial. Brácteas 2 persistentes de hasta $3 \mathrm{~cm}$ de largo aproximadamente, triangulares. Inflorescencia cimosa, glabra, de dicasios consecutivos, pedúnculo de $25 \mathrm{~cm}$ de largo o más, entrenudos de hasta $13 \mathrm{~cm}$ de largo, $6.5 \mathrm{~cm}, 2.5 \mathrm{~cm}$ y así consecutivamente más cortos hasta llegar a las piezas florales. Bracteolas 2 persistentes de $7 \times 5 \mathrm{~mm}$, ovadas. Perianto blanco a rosáceo. Flores masculinas glabras con 4 tépalos: 2 tépalos inferiores $11 \times 15 \mathrm{~mm}$, oblongos; 2 tépalos superiores 14 × $6 \mathrm{~mm}$, ovados. Androceo amarillo formado por un agregado de 50 estambres a más, estambres unidos en la base y/o fusionados hasta las anteras, de hasta $4 \mathrm{~mm}$ de largo. Anteras obovadas. Flores femeninas glabras con 5 tépalos: 2 tépalos inferiores $9 \times 10 \mathrm{~mm}$, oblongos; 3 tépalos superiores $5 \times 8 \mathrm{~mm}$, ovados a oblongos. Gineceo amarillo con 3 estilos, fusionados menos de la mitad, bifurcados una vez, persistentes en el fruto, de hasta $4 \mathrm{~mm}$ de largo, estigma en una banda espiralada alrededor del estilo. Ovario trilocular de hasta $9 \times 8$ $\mathrm{mm}$, alado con 3 prolongaciones blanquecinas a rosadas de 2 $\mathrm{mm}$, de placentación axilar, lóculos biplacentarios, protegido por 3 bractéolas blanquecinas a rosadas. Fruto cápsula, globoso, marrón cuando seco (maduro), trialado.

Material examinado: PERÚ. Lambayeque, provincia de Lambayeque. Distrito Salas. Entre Kerguer y Hualanga, 611'3"S 79²9'57"W, 1450 m, 12 mayo 2017, B. Esquerre

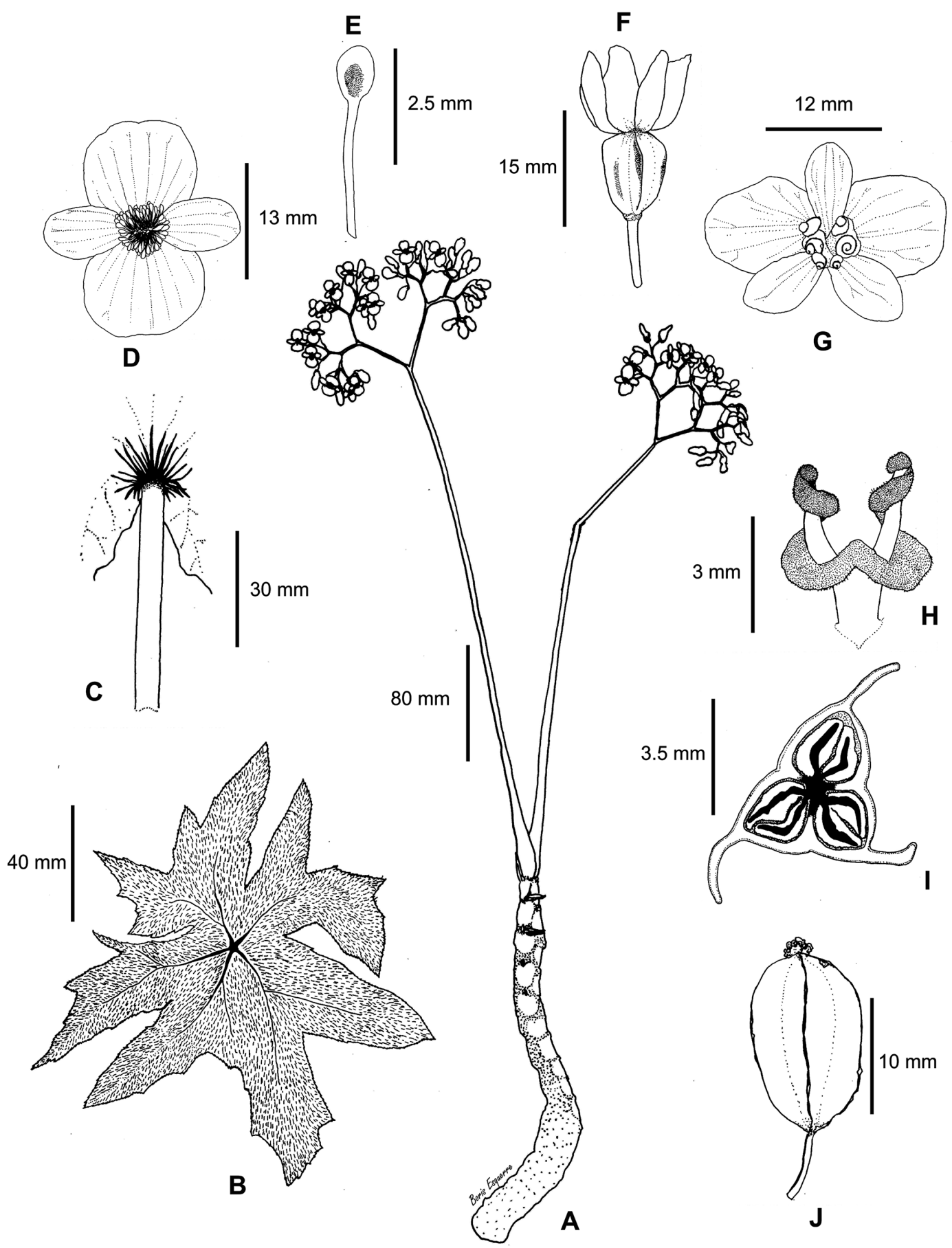

Figura 2. Begonia ludwigii: (A) Tallo e inflorescencia, (B) Hoja, cara adaxial, (C) Peciolo, (D) Flor masculina en antesis. (E) Estambre, (F) Flor femenina y ovario, $(G)$ Flor femenina en antesis $(H)$ Estilo, (I) Transcorte de ovario alado y placentación, (J) Fruto. 
155 (HUT). Distrito Inkawasi. Cerca de Moyán, 6¹7'25"S 79²6'19"W, 1420 m, 10 enero 2018, B. Esquerre 164 (HUT). Piura, provincia de Huancabamba. Distrito Huarmaca. Entrada a Limón de Porcuya, 553'7"S 79³2'4"W, $1541 \mathrm{~m}, 12$ mayo 2017, B. Esquerre 156 (HUT). Cajamarca, provincia de San Miguel. Distrito La Florida. Alrededores de La Florida,6 6 53 '37"S $79^{\circ} 7^{\prime} 8$ "W, 1480 m, fecha?, M.Tebbitt \& A. Daza 846 (MOL).

Begonia ludwigii es una begonia canescente suculenta, perteneciente a la polifilética Sección Knesebeckia, por poseer tallo tipo cańa, con una hilera de tricomas en la unión lámina-peciolo, anteras obovadas y estilos bifurcados, aún persistentes en el fruto péndulo. La característica del anillo de tricomas es especial pues es única de la especie, e inusual dentro de las especies de la Sección Knesebeckia en el Perú. Especies parecidas dentro del Perú no existen, pero sí en otros paises, como por ejemplo Begonia crassicaulis Lindley (Guatemala), B. leathermaniae O’Reilly \& Karegeannes (Bolivia) y B. pachypoda Kollmann \& Peixoto(Brasil).

Durante las expediciones en la zona Amotape-Huancabamba, en el noroeste del Perú, se ha podido hallar individuos de $B$. ludwigii en la zona andina de los departamentos de Lambayeque y Piura (Figura 1). En Piura, se halló una población cercana a una quebrada, en la subida hacia Limón de Porcuya, en el distrito de Huarmaca, se pudo observar unos 16 individuos; en Lambayeque se hallaron dos poblaciones, la primera en el
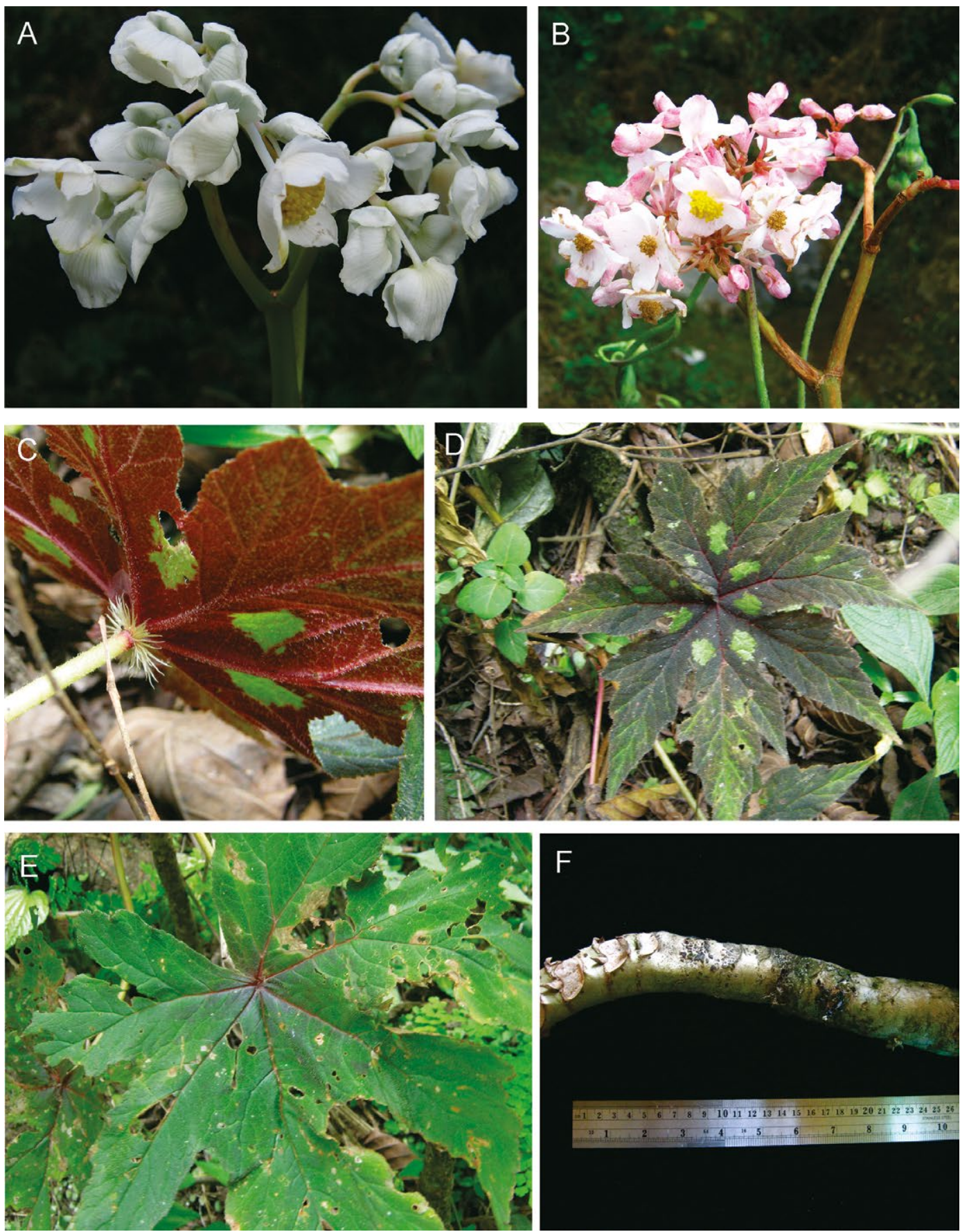

Figura 3. Begonia ludwigii in situ, en territorio peruano: (A) Dpto. Lambayeque, Distrito Salas; flores blancas, (B) Dpto. Piura, Distrito Huarmaca; flores rosado-blanquecinas, (C-D) Dpto. Lambayeque, Distrito Inkawasi; serie de tricomas en la unión peciolo-lámina de hoja juvenil, cara abaxial y adaxial, $(E)$ Dpto. Lambayeque, Distrito Inkawasi; hoja madura, $(F)$ Detalle del tallo suculento y cicatrices peciolares. 
distrito de Salas, en la vía hacia Penachí, registrándose unos 8 individuos al borde del camino, y la segunda cerca de Moyán, en el distrito de Inkawasi, observándose 5 individuos en la ladera de la montańa. Anteriormente se informó que Begonia ludwigii ocurría en Cajamarca, Perú, por Tebbitt (2017), pero en esta publicación se dan pocos detalles de la presencia de la especie en el Perú. Este estudio pretende documentar en mayor detalle el alcance de la ocurrencia conocida de $B$. ludwigii en el Perú.

Recientes trabajos de campo en Ecuador también han llevado al descubrimiento de varias poblaciones nuevas de B. ludwigii, por lo que ahora se sabe que la especie se encuentra en las provincias de Chimborazo, Cañar, El Oro y Loja (Figura 1). La especie, por lo tanto, tiene una distribución continua dentro del sur de Ecuador y el norte de Perú, y se produce en ambos países a lo largo de las estribaciones occidentales de los Andes en un hábitat similar.

Especímenes adicionales examinados.- Begonia ludwigii: ECUADOR. Provincia de Chimborazo, cantón Alausí, antiguo camino entre Huigra y la catarata, $02^{\circ} 19^{\prime} 557^{\prime \prime S} 079^{\circ} 00^{\prime} 421^{\prime \prime W}$, 1380 m, 18 enero 2014, M. Tebbitt, A. Jara \& C. Toapanta 791 (QCA!). Cantón Alausí. Huigra, 1200 m, 4-27 julio 1923, $A$. Hitchcock 20315 (US!). Valle del Río Chanchan, Naranjapata, 530 m, 25 setiembre 1933, Diels 1204 (B!). Norte de Huigra, 1524-1980 m, 19-28 mayo 1945, W. Camp E-3317 (K!, NY!). Entre Alausí y El Triunfo, 19 julio 1986, T. Croat 61575 (MO!). Cantón Bucay. Pallatanga. 32 km desde Pallatanga, 650 m, 29 agosto 1976, B. Øllgaard \& H. Balslev 9007 (AAU!, MO!). Provincia de Cañar, cantón La Troncal. Manta Real. Carretera de Manta Real a Caimatán km 6, 28 mayo 2005, H. Vargas \& W. Defas 5707 (QCNE!, MO!). Provincia de El Oro, cantón Zaruma, camino sobre Zaruma, sombreado, acantilado húmedo al lado de una pequeña corriente, 03³9'409"S 079³3'946"W, 930 m, 16 January 2014, M. Tebbitt, A. Jara \& C. Toapanta 780 (QCA!). Cantón Pińas. Reserva Buenaventura, 1000 m, 1 diciembre 2006, L. Jost \& N. Simpson 8431 (QCA!). Cantón Balsas, antiguo camino de Balsas a Pińas, banco de tierra abierto en la carretera, $03^{\circ} 44.213^{\prime} \mathrm{S}, 79^{\circ} 48.114^{\prime} \mathrm{W}, 1126 \mathrm{~m}, 25$ mayo 2016, M. Tebbitt \& D. Gutierrez 863 (QCA!).

En el lado peruano, las poblaciones registradas se encuentran sin protección, en áreas degradadas por el avance urbano, construcción de carreteras y colonización rural desordenada, por lo que se les considera amenazadas. Además en las poblaciones de Lambayeque se han registrado incendios forestales intencionales en los alrededores, lo que amenaza estas poblaciones. Es probable que por el por el tipo de hábitat en que se encuentra distribuida esta especie, en el noroeste del Perú, se halle representada dentro de algún área de protección silvestre nacional (SINANPE) o privada, por ejemplo dentro del Área de Conservación Regional Moyán-Palacio y Refugio de Vida Silvestre Laquipampa (Lambayeque), Área de Conservación Regional Bosques Secos Salitral-Huarmaca (Piura) y Refugio de Vida Silvestre Bosques Nublados de Udima (Cajamarca), por ello son necesarias colectas y registros en aquellas zonas para confirmar su protección. En Ecuador, se le ha catalogado como especie Vulnerable (VU) de acuerdo al Libro Rojo de Endemismos Ecuatorianos (León-Yáñez et al. 2011), por lo tanto, siguiendo los lineamientos propuestos por la Unión Internacional para la Conservación de la Naturaleza (IUCN 2013) en el Perú se propone considerar a $B$. ludwigii también como especie Vulnerable (VU) hasta que más colectas y registros confirmen que este taxón se encuentre al menos en una de las zonas de protección silvestre mencionadas anteriormente. La floración se reporta de abril a agosto.

\section{Begonia parcifolia C. DC.}

(Figs. 4-6)

Smithsonian Miscellaneous Collections 69(12): 10, 1919

Tipo: ECUADOR, Provincia de Loja, Cariamanga, 24 Noviembre 1910, Townsend 947 (holotipo US!).

Begonia nervidens Irmsch., Bot. Jahrb. Syst., 74: 614, 1949. TIPO: ECUADOR, Provincia de El Oro, entre Portovelo Mina de oro cerca de Zaruma) y El Tambo, alt. 600 a 1000 m, 2 Setiembre 1923, Hitchcock 21279 holotipo: US!).

Hierba acaulescente, tuberosa; hojas caducas, que se desarrollan justo antes o al mismo tiempo que las inflorescencias. Tubérculo ampliamente elipsoide a esferoide con una depresión superficial en la superficie superior, a menudo con un tronco floral subterráneo corto de $10 \mathrm{~cm}$ de largo que porta hojas en su ápice y a veces también en una rama lateral corta hasta ca. 0.5 cm de largo, tubérculo ca. $5.5-12 \mathrm{~cm}$ de diámetro. Estípulas persistentes, ovadas a ovadas triangulares, $0.5-1.4 \times 0.25-0.75$ $\mathrm{cm}$, ápice agudo a obtuso, apiculado, margen entero a ciliado. Hojas 2 a 6, alternas, basifijas; pecíolo $3.5-25 \mathrm{~cm}$ de largo, tomentoso, tricomas ca. $2 \mathrm{~mm}$; hoja asimétrica, ampliamente ovalada, o subreniforme a suborbicular, $3-18.5 \times 4-13.5 \mathrm{~cm}$, ápice usualmente abruptamente agudo o abruptamente acumi-

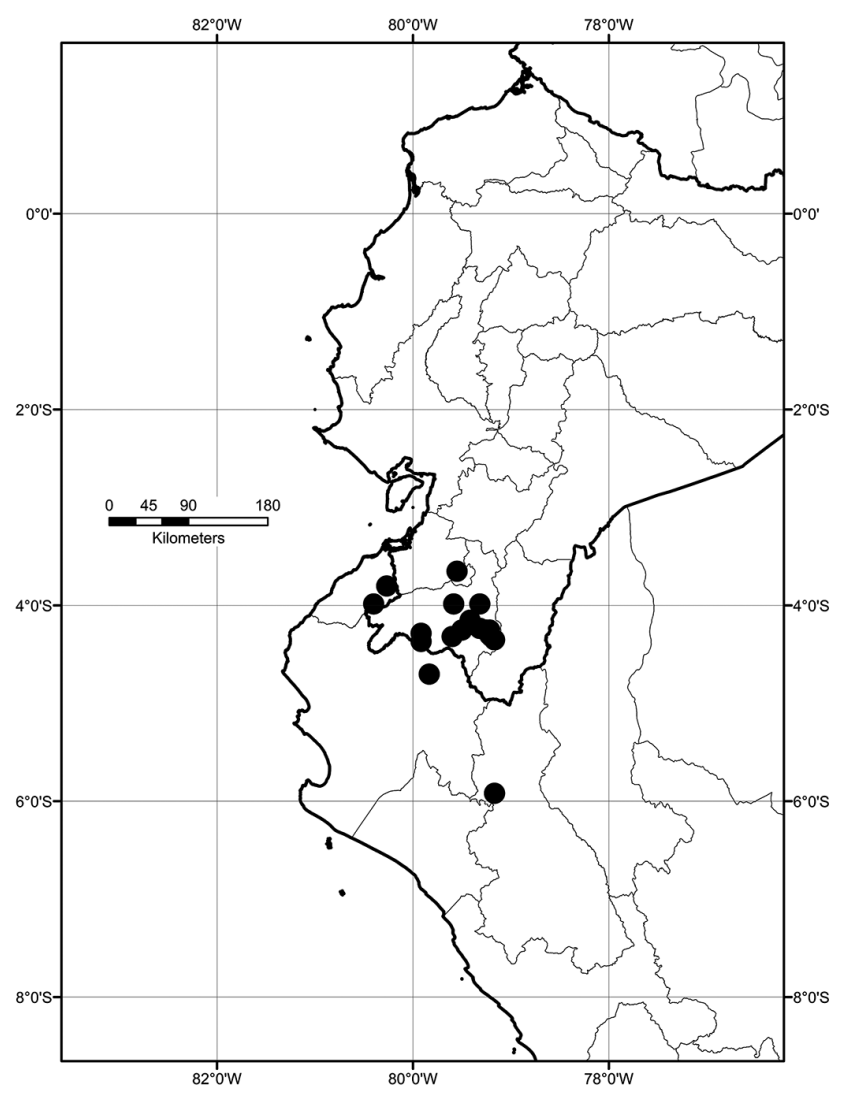

Figura 4. Distribución de Begonia parcifolia en Ecuador y Perú. 
nado, base oblicuamente cordada, lóbulos basales extendidos pero usualmente superpuestos hasta la mitad de su longitud, seno $1.3-6 \mathrm{~cm}$ de profundidad, margen con lóbulos irregulares cortos más o menos triangulares o sin ellos, lóbulos de $2 \mathrm{~cm}$ de profundidad cuando presentes, margen dentado, dientes de 0.25-1.75 mm de largo, ciliados, ca. $1 \mathrm{~mm}$ de grosor y bastante coriáceos cuando la planta emerge de la latencia pero a menudo se vuelve más delgada y jugosa en la madurez, cara adaxial verde pálido, cara abaxial blanquecino-verde, ambas caras escasas de densa vellosidad blanca, venas palmadas 7 a 10 . Inflorescencias 1 a 4, axilares, que surgen de la porción superior del tubérculo o corto tronco floral subterráneo, más o menos erectas, asimétricas a simétricas, cimas dicasiales, con una a cuatro ramas, llevando pocas o varias flores en un momento dado, flores masculinas generalmente protandras, pero ambos sexos se abren concurrentemente; pedúnculo de $15-45 \mathrm{~cm}$ de largo, escaso de densa vellosidad; pedicelos de flores masculinas de 0.25-2.5 $\mathrm{cm}$ de largo, con tricomas como los del pedúnculo; pedicelos

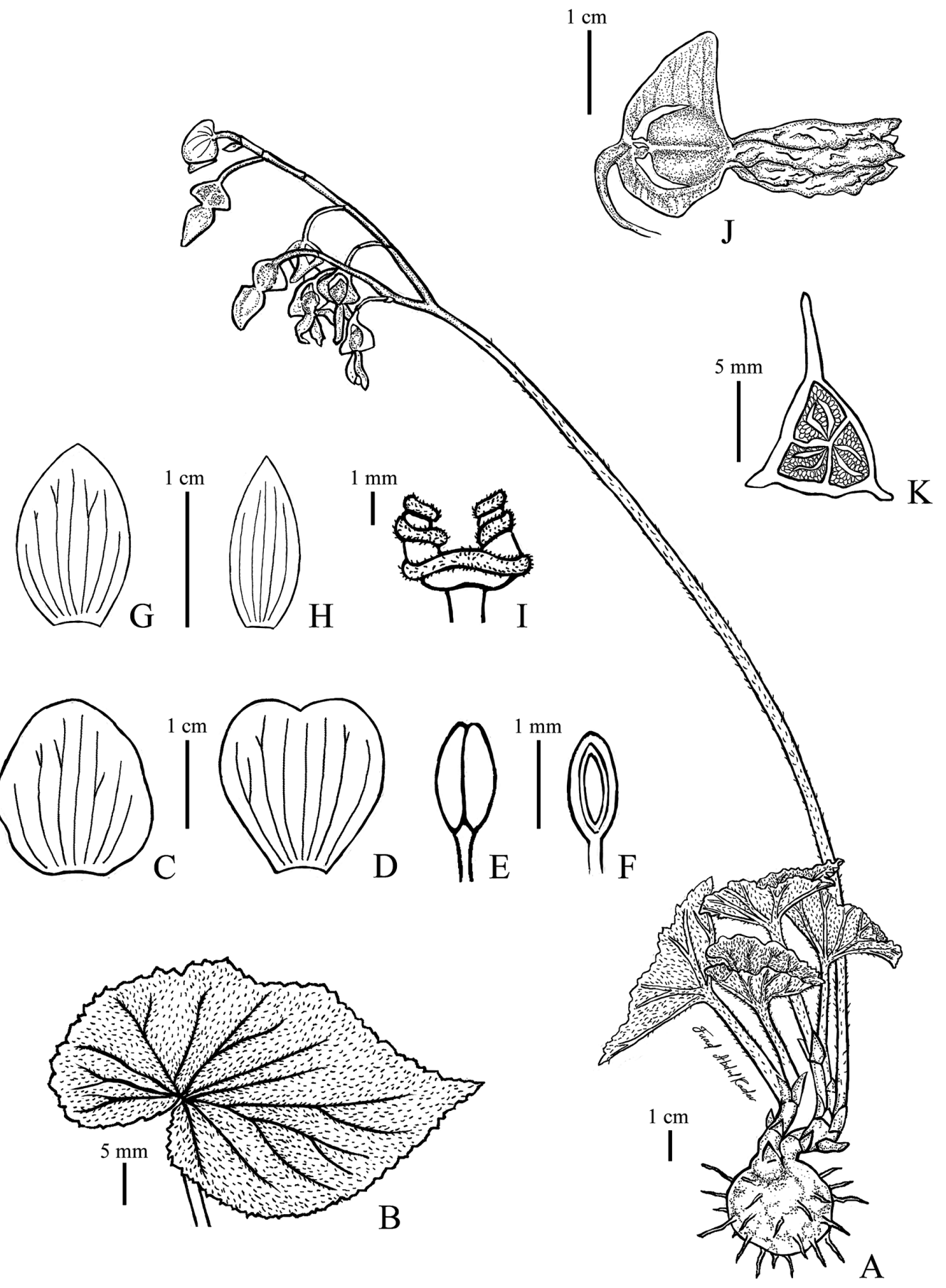

Figura 5. Begonia parcifolia: (A) Hábito, planta en floración con hojas jóvenes. (B) Hoja madura, hoja aplanada para mostrar el contorno. (C) Tépalo inferior masculino. (D) Tépalo superior masculino. (E) Estambre, vista frontal. (F) Estambre, vista lateral. (G) Tépalo inferior femenino. (H) Tépalo superior femenino. (I) Estilo. (J) Fruta madura dehiscente. (K) Ovario, sección transversal (Dibujado por Fuad Abdulkader, basado en Tebbitt 769). 
de flores femeninas de $0.3-3 \mathrm{~cm}$ de largo, con pelos como los del pedúnculo; brácteas caducas, ovadas, romboides-ovadas, oblongas u oblongas-obovadas, $0.4-1.5 \times 0.2-0.85 \mathrm{~cm}$, ápice obtuso a agudo, margen fimbriado a denticulado-fimbriado con tricomas uniseriados no glandulares $1.5 \mathrm{~mm}$ de largo, superficies glabras. Flores masculinas con 4 tépalos, extendidos: 2 tépalos inferiores rosados por fuera y blanco a rosa pálido en el interior, estrechamente obovados u oblongo-elípticos, $0.8-3 \times 0.7-1.6$ $\mathrm{cm}$, ápice obtuso, margen entero, glabro o casi glabro; 2 tépalos superiores blanco o rosa pálido en ambas caras, ampliamente obovados, $1.4-2.4 \times 0.9-2.2 \mathrm{~cm}$, ápice con una muesca superficial, margen entero, glabro; androceo en un agregado de 50 a 100 o más estambres, unidos a un receptáculo plano o a un torus de 1-4 $\mathrm{mm}$ de alto, filamentos de 2-6 $\mathrm{mm}$ de largo, aquellos cerca del centro del androceo más largos que aquellos en el margen, anteras simétricamente basifijas, elipsoidales, cuboides u obovoides, 1-1.5 mm de largo, dehiscencia a través de hendiduras unilaterales, conectivos no extendidos. Flores femeninas con 5 tépalos, bractéolas ausentes; tépalos persistentes, extendidos: 2 tépalos inferiores rosados, rosado y blanco a rosado pálido en el interior, obovado-elípticos a elípticos, u ovado-oblongos a obovado-oblongos, ápice obtuso; 3 tépalos superiores blancos o rosado pálidos en ambas caras, obovados a ampliamente obovados, subiguales, $0.6-2 \times 0.4-1.4 \mathrm{~cm}$, ápice más o menos truncado, margen entero, glabro; ovario ampliamente obovoide o ampliamente elipsoide a subesférico, 3-11 × 2-9.5 mm, verde a rojo, glabro, alado con tres prolongaciones, un ala más larga que las otras dos, triangular redondeada, borde frontal del ala más larga más o menos truncado o convexo, curvada hacia atrás aproximadamente a la mitad del largo del ovario, borde posterior suavemente convexo-curvado, ápice subagudo a obtuso, $0.1-1.1 \mathrm{~cm}$ de largo, $0.5-1,5 \mathrm{~cm}$ de ancho, dos alas más cortas triangulares, 1-5 mm de largo, 5-15 mm de ancho, ápice obtuso; trilocular; estilos 3, brevemente fusionados en la base,
3-6.5 mm de largo, bífidos desde aproximadamente la mitad de su altura, ramas erectas, papilas estigmáticas en una banda una vez retorcida en espiral. Pedúnculo fructífero de 27.5-38 $\mathrm{cm}$ de largo; pedicelo fructífero que se alarga brevemente y se curva ligeramente hacia abajo en toda su longitud, hasta $3 \mathrm{~cm}$ de largo. Fruto casi erecto a péndulo, elipsoide a casi esferoide, $1.1 \times 1.2 \mathrm{~cm}$, alas glabras, ala más larga $1.5 \mathrm{~cm}$ largo x $1.7 \mathrm{~cm}$ ancho, ápice generalmente obtuso, ocasionalmente agudo, alas más cortas $0.8 \mathrm{~cm}$ largo x $1.7 \mathrm{~cm}$ ancho, ápice generalmente obtuso, ocasionalmente agudo.

Material examinado: PERÚ. Tumbes, provincia de Zarumilla, Pampas de Hospital, entre el cruce al puesto de vigilancia Cabo Cotrina y el Caucho, ca. 359'S 80²4'W, $450 \mathrm{~m}$, 23 octubre 1988, C. Díaz \& R. Vásquez 3115 (MO!). Piura, provincia de Ayabaca, camino entre Las Lomas y Ayabaca, inmediatamente sobre el puente Tondopa, $4^{\circ} 42^{\prime} \mathrm{S} 79^{\circ} 50^{\prime} \mathrm{W}, 955-$ 1627 m, 29 mayo 2015, M. Tebbitt \& A. Daza 845 (E!, MOL!). Cajamarca, Jaén, Colasay, Bosque seco, $05^{\circ} 55^{\prime} \mathrm{S} 79^{\circ} 10^{\prime} \mathrm{W}, 1600$ m, 8 diciembre 2001, R. Vásquez, R. Rojas \& L. Campos 27225 (HUT!, MO!, USM!).

Begonia parcifolia perteneciente también a la Sección Knesebeckia se reconoce fácilmente por su hábito acaulescente, presencia de tubérculos, tépalos femeninos que persisten en los frutos y estilos bífidos. La especie es inusual entre las begonias tuberosas por desarrollar en sus tubérculos troncos florales de hasta $10 \mathrm{~cm}$ de largo cuando crecen en hábitats inestables.

Begonia parcifolia es una especie dispersa y localmente común del sur de Ecuador (provincias El Oro y Loja) y ahora el noroeste del Perú (departamentos Piura y Cajamarca). Ocurre en laderas de lutitas semiáridas, escasamente escarpadas, escasamente pobladas, entre matorrales o bosque caducifolio, a menudo en acantilados húmedos y sombreados junto a arroyos y ríos, de 600 a $2200 \mathrm{~m}$. La floración se reporta de setiembre a febrero.

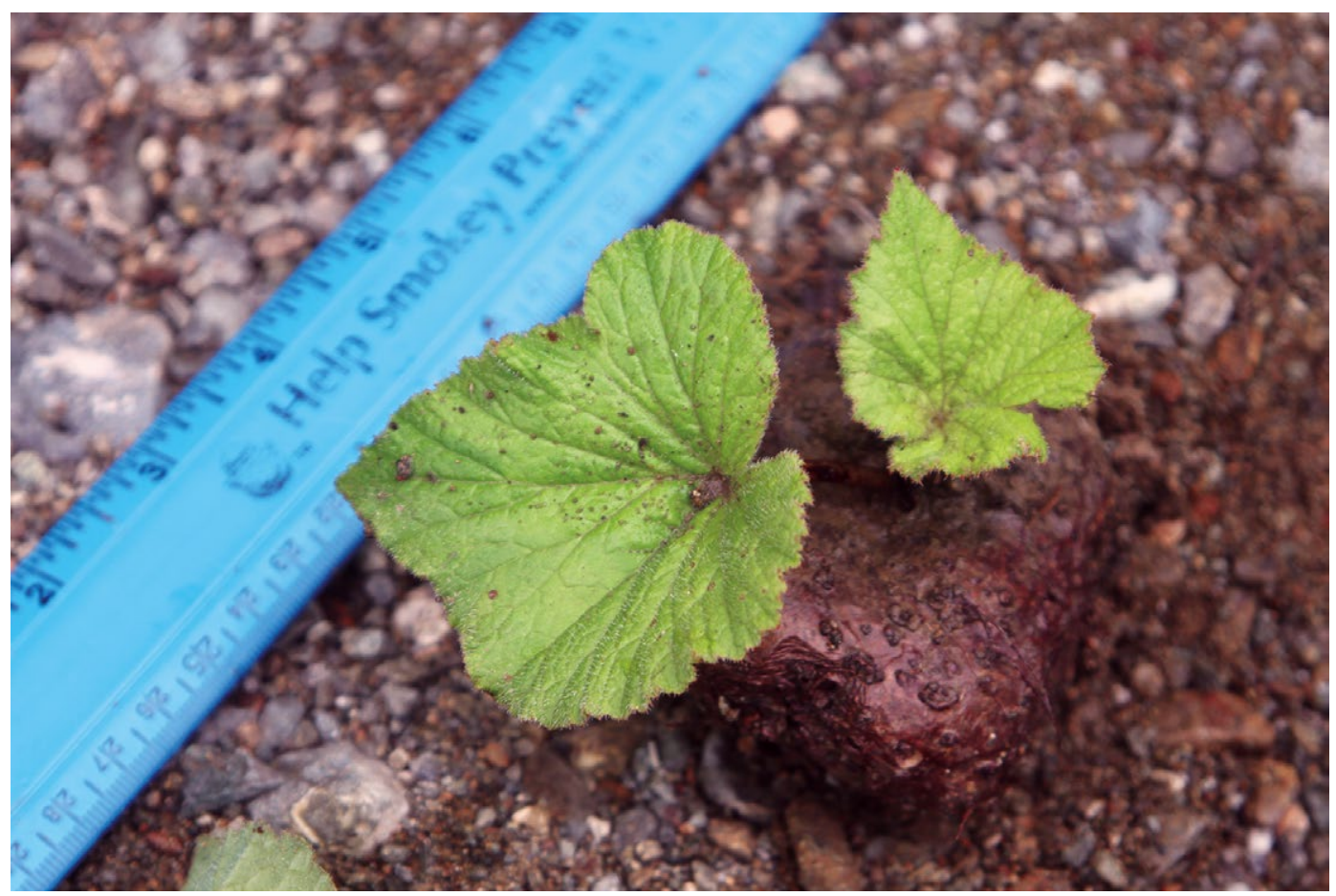

Figura 6. Begonia parcifolia in situ, en territorio peruano: Dpto. Piura, Provincia Ayabaca; tubérculo y hojas (Tebbitt \& Daza 845). 
Especímenes adicionales examinados: Begonia parcifolia: ECUADOR. Provincia de El Oro, cantón Portovelo, entre Portovelo (Mina de oro cerca a Zaruma) y El Tambo, 600-1000 $\mathrm{m}$, setiembre 1923, A. Hitchcock 21301 (US!); cercanías de Portovelo, 6-15 octubre 1918, J. Rose 23394 (US); Puente Negro cerca a Porto Velo, 914 m, 30 noviembre 1948, N. B. Foster 2603 (US!); Cantón Zaruma, camino sobre Zaruma, $3^{\circ} 39^{\prime} \mathrm{S} 79^{\circ} 33^{\prime} \mathrm{W}$, 930 m, 16 enero 2014, M. Tebbitt 779 (QCA!). Provincia de Loja, entre Loja y Portovelo, 3-6 octubre 1918, J. N. Rose 23346 (US!), 23364 (US!); cantón Chaguarpamba, entre Portovelo y Loja, cruzando el puente del Río Puyango, $3^{\circ} 45^{\prime} \mathrm{S} 79^{\circ} 38^{\prime} \mathrm{W}$, 602 m, 16 enero 2014, M. Tebbitt 783 (QCA!, MO!), 350'S 79³4'W, 973 m, 16 enero 2014, M. Tebbitt 784 (QCA!, MO!), $3^{\circ} 53^{\prime} \mathrm{S} 79^{\circ} 33^{\prime} \mathrm{W}, 1072 \mathrm{~m}, 16$ enero 2014, M. Tebbitt 785 (QCA!, MO!); después del puente pasando Yangana, $4^{\circ} 21^{\prime} \mathrm{S} 79^{\circ} 10^{\prime} \mathrm{W}$, ca. 1900 m, 19 enero 1997, R. Pennington, V. Plana, G. Lewis \& K. Klitgaard-Lewis 661 (E!, QCA!, QCNE!). Cantón Catamayo, Camino a La Toma, ca. km 5 desde La Toma, 359'S 79¹9'W, 1600-1800 m, 26 noviembre 1985, J. Madsen 61158 (AAU!, QCA!, QCNE!); $24 \mathrm{~km}$ suroeste de Chinchas sobre el camino a Catacocha, $3^{\circ} 59^{\prime} \mathrm{S} 79^{\circ} 35^{\prime} \mathrm{W}, 1860 \mathrm{~m}, 3$ febrero 1979, R. King \& F. Almeda 7960 (US!); $27-30 \mathrm{~km}$ al suroeste de Catamayo en la vía a Cariamango, $4^{\circ} 9^{\prime} \mathrm{S} 79^{\circ} 25^{\prime} \mathrm{W}, 1920 \mathrm{~m}, 2$ febrero 1979 , R. King \& F. Almeda 7943 (US!); cantón Loja, cercanías a Vil-

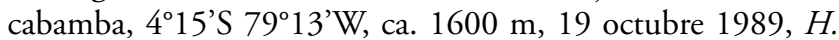
Luther et al 2746 (SEL!); oeste de Vilcabamba, $4^{\circ} 17^{\prime} \mathrm{S} 79^{\circ} 13^{\prime} \mathrm{W}$, ca. 2000 m, 26 noviembre 1981, J. Madsen et al. 36744 (AAU!, QCA!); cantón Gonzanamá, Carretera Vilcabamba - Yangana Toledo, $4^{\circ} 15^{\prime} \mathrm{S} 79^{\circ} 15^{\prime} \mathrm{W}, 2000$ m, 22 diciembre 1991, D. Rubio, E. Aulestia \& K. Edwards 2275 (MO!, QCA!, QCNE!); Camino entre Gonzanamá y Cariamanga, 4 $15^{\prime} \mathrm{S} 79^{\circ} 30^{\prime} \mathrm{W}, 1768 \mathrm{~m}$, 14 enero 2014, M. Tebbitt 769 (QCA!); Cantón Macará, ruta E35 ca. $12 \mathrm{~km}$ norte de Macará, 4 $17^{\prime} \mathrm{S} 79^{\circ} 55^{\prime} \mathrm{W}, 1170 \mathrm{~m}, 14$ enero 2014, M. Tebbitt 771 (QCA!); Caracas entre Vilcabamba y Yangana, $4^{\circ} 18^{\prime} \mathrm{S} 79^{\circ} 13^{\prime} \mathrm{W}, 1900-2000 \mathrm{~m}, 29$ octubre 1984, $P$. Jørgensen 56281 (AAU!); camino antiguo Vilcabamba-Yangana, sur de Solanda, colinas sobre el río Solanda, $4^{\circ} 19^{\prime} \mathrm{S} 79^{\circ} 12^{\prime} \mathrm{W}$, ca. 1600 m, 14 febrero 1993, G. Harling \& B. Ståhl 26393 (S!); lado noreste del Cerro Mataperro, $3 \mathrm{~km}$ suroeste San Pedro, 10 $\mathrm{km}$ WNW de Catamayo, 4²19'S 79³6'W, 1815 m, 10 enero 1945, F. Fosberg \& M. Giler 22944 (US!).

Begonia parcifolia tiene una distribución dispersa a través de su rango y a menudo ocurre en pequeñas poblaciones que constan de menos de cien plantas. Varios de los sitios conocidos están situados junto a caminos no pavimentados y podrían verse afectados adversamente por la futura ampliación de la carretera. No se conoce que la especie se encuentre en Ecuador o Perú dentro de un área protegida. En consecuencia, evaluamos a $B$. parcifolia como Vulnerable (VU) según los criterios de la UICN (IUCN 2013).

\section{Agradecimientos}

A la Universidad Nacional Pedro Ruiz Gallo por facilitar la movilidad a los sitios de colecta para Begonia ludwigii. A los curadores de los herbarios AAU, E, HUT, MO, MOL, QCA, QCNE, S, SEL, US y USM por permitirnos consultar el material de su colección. A Consuelo Rojas, José Ayasta, Luciana Alarcón, Gabriela Torres (Perú), Aniceto Daza (MOL), Adolfo Jara (ANDES), Cristina Toapanta (QCA) y David Gutiérrez (Ecuador) por su compañía y ayuda prestada en las colectas de campo. A Guillermo Delgado por permitir el estudio morfológico de B. ludwigii en el Laboratorio de Cultivo de Tejidos Vegetales y Recursos Fitogenéticos de la Universidad Nacional Pedro Ruiz Gallo en Lambayeque, Perú. Agradecemos a Fuad Abdulkader por el dibujo de Begonia parcifolia.

\section{Literatura citada}

Brako L \& J. Zarucchi. 1993. Catálogo de plantas con flores y gimnospermas del Perú. Monographs in Systematic Botany 45. Missouri Botanical Garden, St. Louis, MO.

Doorenbos J.; M. Sosef \& J. De Wilde. 1998. The sections of Begonia including descriptions, keys and species lists (Studies in Begoniaceae VI). Wageningen Agricultural University Papers 98(2): 1-266.

Esquerre-Ibañez B. 2015. Passiflora dorisiae, una nueva especie en el subgénero Passiflora (Passifloraceae). Revista Peruana de Biología 22(3): 303-308. http://dx.doi.org/10.15381/rpb. v22i3.11435

IUCN. 2013. Guideline for using the IUCN Red List Categories and Criteria. Version 10. Prepared by the Standards and Petitions Subcommittee. Downloadable from http://www.iucnredlist. org/documents/RedListGuidelines.pdf.

León B.; J. Roque \& C. Ulloa Ulloa. 2006. Libro Rojo de las Plantas Endémicas del Perú. Revista Peruana de Biología, Número Especial 13(2): 1-971.

León-Yáñez S.; R. Valencia; N. Pitman; L. Endara; C. Ulloa Ulloa \& H. Navarrete. 2011. Libro Rojo de las Plantas Endémicas del Ecuador, Segunda Edición. Publicaciones del Herbario QCA. Pontificia Universidad Católica del Ecuador.

Moonlight P.; C. Reynel \& M.C. Tebbitt. 2017. Begonia elachista Moonlight \& Tebbitt sp. nov., an enigmatic new species and a new section of Begonia (Begoniaceae) from Peru. European Journal of Taxonomy 281: 1-13. https://doi.org/10.5852/ ejt.2017.281

Moonlight P. \& M.C. Tebbitt. 2017. Two new Peruvian species of Begonia (Begoniaceae) and an amended description of Begonia thyrsoidea. Edinburgh Journal of Botany 74(2):111-122. https://doi.org/10.1017/S0960428616000299

Rajbhandary S. 2013. Traditional uses of Begonia species (Begoniaceae) in Nepal. Journal of Natural History Museum 27: 25-34. http://dx.doi.org/10.3126/jnhm.v27i0.14150

Rodríguez E. \& R. Rojas. 2002. El herbario, administración y manejo de colecciones botánicas. Herbarium Truxillense. Jardín Botánico de Missouri-Perú. Pasco. 31 pp.

Tebbitt M.C. 2005. Begonias: Cultivation, identification and natural history. Timber Press. 272 pp.

Tebbitt M.C. 2011. A new and unusual xerophytic species of Begonia (Begoniaceae) from Peru. Edinburgh Journal of Botany 68(2):1-6. https://doi.org/10.1017/S0960428611000096

Tebbitt M.C. 2015. Two new species of Andean tuberous Begonia in the Begonia octopetala group (Begoniaceae). Novon 23: 479-489. http://doi.org/10.3417/2013027

Tebbitt M.C. 2016. Two new species of Andean Begonia (Begoniaceae). Edinburgh Journal of Botany 73(1):143-152. https://doi. org/10.1017/S0960428615000335

Tebbitt M.C, 2017. Recircumscription and new synonyms of Begonia acerifolia (Begoniaceae) and amended descriptions of the poorly known B. hydrophylloides and B. velata. Edinburgh Journal of Botany 74(2):217-228. http://doi.org/10.1017/ S0960428617000105

TROPICOS. Missouri Botanical Garden. 04 Feb $2018<$ http://www. tropicos.org/Name/3400758>

Ulloa C.; J. Zarucchi \& B. León. 2004. Diez años de adiciones a la Flora del Perú: 1993-2003. Arnaldoa (edición especial): 7-242. http://dx.doi.org/10.5962/bhl.title.63538

Weigend M. 2002. Observations of the biogeography of the AmotapeHuancabamba zone in Northern Peru. The Botanical Review 68(1):38-54. http://dx.doi.org/10.1663/00068101(2002)068[0038:OOTBOT]2.0.CO;2 